\title{
Postnatal growth restriction augments oxygen-induced pulmonary hypertension in a neonatal rat model of bronchopulmonary dysplasia
}

Stephen Wedgwood', Cris Warford', Sharleen C. Agvateesiri', Phung Thai' ${ }^{2}$, Sara K. Berkelhamer ${ }^{3}$, Marta Perez ${ }^{4}$, Mark A. Underwood' and Robin H. Steinhorn ${ }^{5}$

BACKGROUND: Prematurity and fetal growth restriction are risk factors for pulmonary hypertension $(\mathrm{PH})$ in infants with bronchopulmonary dysplasia (BPD). Neonatal rats develop $\mathrm{PH}$ and vascular remodeling when exposed to hyperoxia. We hypothesize that postnatal growth restriction (PNGR) due to under-nutrition increases the severity of $\mathrm{PH}$ induced by hyperoxia in neonatal rats.

METHODS: Pups were randomized at birth to litters maintained in room air or 75\% oxygen (hyperoxia), together with litters of normal milk intake (10 pups) or PNGR (17 pups). After $14 \mathrm{~d}$, right ventricular hypertrophy (RVH) was assessed by Fulton's index (right ventricular weight/left ventricular plus septal weight) and PH by echocardiography. Lungs were analyzed by immunohistochemistry, morphometrics, western blotting, and metabolomics.

RESULTS: Hyperoxia and PNGR each significantly increased pulmonary arterial pressure, RVH and pulmonary arterial medial wall thickness, and significantly decreased pulmonary vessel number. These changes were significantly augmented in pups exposed to both insults. Hyperoxia and PNGR both significantly decreased expression of proteins involved in lung development and vasodilation.

CONCLUSION: PNGR induces right ventricular and pulmonary vascular remodeling and augments the effects of oxygen in neonatal rats. This may be a powerful tool to investigate the mechanisms that induce PH in low-birth-weight preterm infants with BPD.

B ronchopulmonary dysplasia (BPD) is a serious and common chronic lung disease of infancy (1), affecting up to one-third of extremely preterm infants and resulting in 10,000 new cases each year in the United States. Recent evidence indicates that up to $18 \%$ of all extremely low-birth-weight infants will develop some degree of pulmonary hypertension (PH), increasing to $25-40 \%$ of infants with moderate to severe BPD (2). Furthermore, recent studies have determined that
$\mathrm{PH}$ contributes to worse clinical outcomes, and significantly increases the morbidity and mortality of preterm birth (3). However, pulmonary hypertension is often not diagnosed in premature infants until symptoms emerge after many weeks of life, when the disease is advanced and associated with severe right ventricular cardiac dysfunction (4). Early screening echocardiograms have not proven to be reliable predictors of PH (5), and no reliable biomarkers yet exist. Recent data established that fetal growth restriction is a significant predictor of $\mathrm{PH}$ at 36 wk corrected gestation (6).

Postnatal growth restriction (PNGR) is very common in preterm infants. After birth, preterm infants lose weight initially and then often grow more slowly than the in utero rate $(7,8)$. The most immature infants are particularly vulnerable, with those born at $\leq 28 \mathrm{wk}$ gestation only achieving $~ 70 \%$ of their expected weight by discharge (7). While PNGR has been associated with worse neurodevelopmental outcomes and higher readmission rates, its effects on cardiopulmonary development are not well understood.

Rodent models have been used to identify the underlying mechanisms that give rise to $\mathrm{PH}$ and BPD in preterm infants. Neonatal rats and mice are born with developmentally immature lungs that are structurally similar to premature infants born at 24 to $28 \mathrm{wk}$ of gestation (9). Exposure of pups to hyperoxia (60\% oxygen and greater) leads to pulmonary hypertension, right ventricular hypertrophy, pulmonary vascular remodeling, and alveolar simplification (10), similar to human infants with PH and BPD.

The aim of this study was to determine whether PNGR increases the susceptibility to $\mathrm{PH}$ in preterm infants, to gain mechanistic insights, and to identify potential diagnostic markers. An established rat model of PNGR involves increasing the number of newborn pups per dam (11). Compared to a standard litter size of 10 pups, a pup litter size of 16 leads dams to produce more milk without alterations in milk lactose or protein contents, but significantly decreases milk fat content. The result is decreased weight gain and both fat and protein

'Department of Pediatrics, UC Davis Medical Center, Sacramento, California; ${ }^{2}$ Department of Internal Medicine, Division of Cardiovascular Medicine, UC Davis Health System, Sacramento, California; ${ }^{3}$ Department of Pediatrics, University at Buffalo, Buffalo, New York; ${ }^{4}$ Department of Pediatrics, Northwestern University, Chicago, Illinois; ${ }^{5}$ Department of Hospitalist Medicine, Children's National Health System, Washington, DC. Correspondence: Stephen Wedgwood (swedgwood@ucdavis.edu) 
a

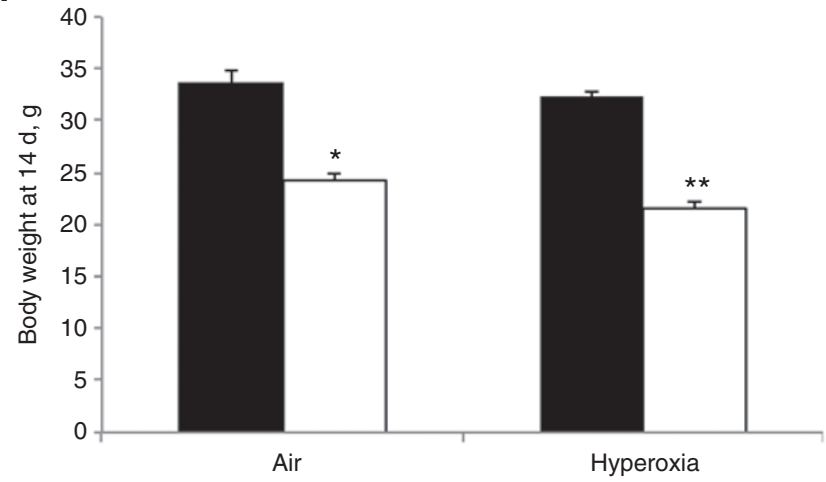

b

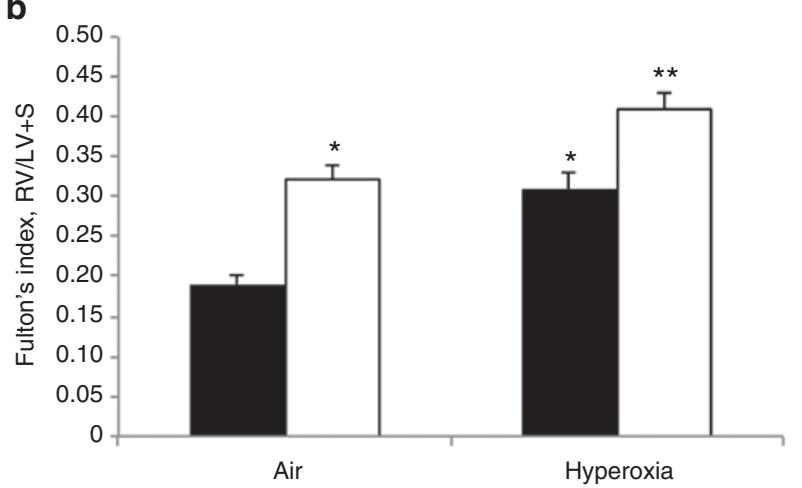

C

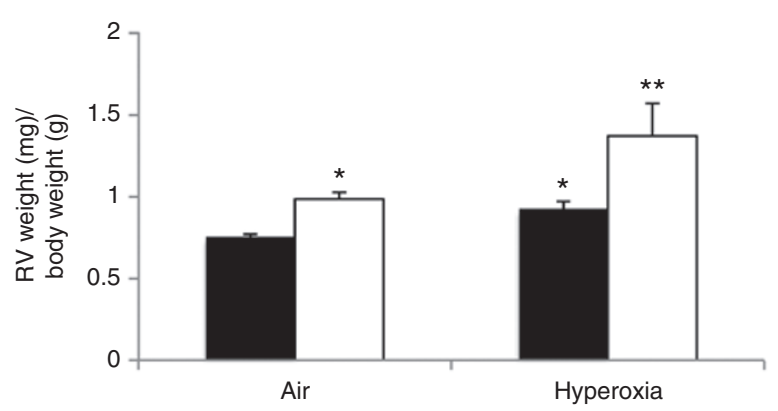

d

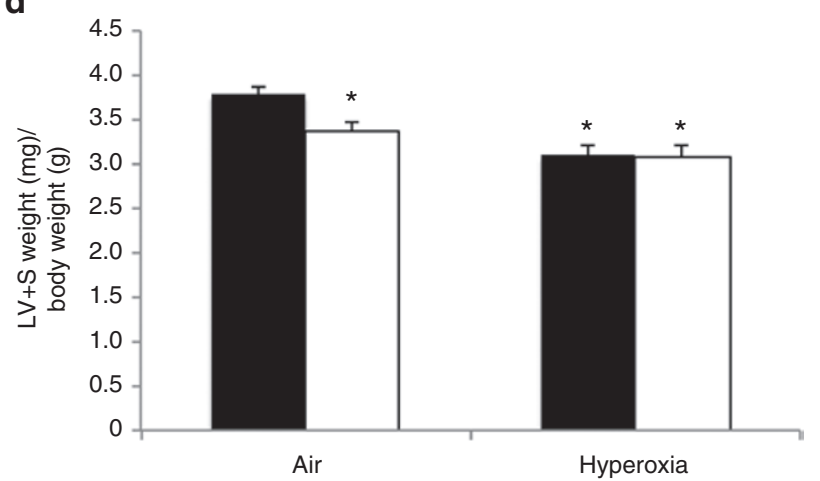

Figure 1. Postnatal growth restriction and hyperoxia induce right ventricular hypertrophy at day 14. Data from control litters are depicted by black columns; data from restricted litters are depicted by white columns. (a) Body weight at day 14 . $N \geq 18$ animals per group. (b) Fulton's index was determined as the weight of the right ventricle divided by the weight of the left ventricle plus septum. (c) The ratio of right ventricular weight to body weight was determined for each animal. (d) The ratio of left ventricular plus septal weight to body weight was determined for each animal. $N \geq 12 \mathrm{hearts}$ per group. ${ }^{*} P<0.05$ vs. room air control. ${ }^{* *} P<0.05$ vs. hyperoxia control.

accretion in the pups. We hypothesized that PNGR would exacerbate the PH and BPD phenotypes evident in the established rat hyperoxia model. To test this hypothesis, we randomized newborn rats to litters of 10 or 17 pups and maintained them in room air or in a chamber exposed to $75 \%$ oxygen for $14 \mathrm{~d}$. We then monitored $\mathrm{PH}, \mathrm{RVH}$, vascular remodeling, lung morphometry and lung gene expression.

\section{RESULTS}

After $14 \mathrm{~d}$, pups subjected to PNGR in room air weighed $24.2 \pm 0.7 \mathrm{~g}$ and control pups weighed $33.7 \pm 1.1 \mathrm{~g}$ (Figure 1, Supplementary Figure S1 online). Fourteen days exposure to $75 \% \mathrm{O}_{2}$ (hyperoxia) had no significant effect on body weight in control pups but did significantly decrease body weight further in growth restriction pups $(21.6 \pm 0.6 \mathrm{~g})$ (Figure 1a). Consistent with previous studies, hyperoxia increased Fulton's index relative to room air controls (10) (Figure 1b). PNGR increased Fulton's index similar in severity to hyperoxia alone, and PNGR combined with hyperoxia caused a significantly greater increase relative to oxygen exposure alone (Figure $1 \mathrm{~b}$ ). We examined whether the changes in Fulton's index were produced solely by right ventricular hypertrophy. PNGR and hyperoxia significantly increased right ventricular weight normalized to body weight, and combined exposure had a greater effect than either insult alone (Figure 1c). We also found that
PNGR and hyperoxia significantly decreased left ventricular weight normalized to body weight, although combined exposure did not produce any further decrease (Figure 1d).

The effects of PNGR and hyperoxia on cardiopulmonary hemodynamics were determined by echocardiography. The ratio of the pulmonary acceleration time to total ejection time (PAT/ET) decreases with increased pulmonary artery pressures, and PNGR and hyperoxia both significantly decreased $\mathrm{PAT} / \mathrm{ET}$ relative to room air controls, with a further significant decreased when both insults were combined (Figure 2).

In agreement with previous studies, hyperoxia significantly increased medial wall thickness of small pulmonary arteries and decreased the number of small pulmonary vessels relative to room air controls (10) (Figure 3). PNGR in room air mimicked the effects of hyperoxia, and a combination caused a more severe phenotype (Figure 3).

Similar to previous reports (10), hyperoxia increased mean alveolar area and decreased radial alveolar count. PNGR did not affect either measure of lung alveolarization and did not accentuate the effect of hyperoxia (Figure 4).

We found that PNGR or hyperoxia substantially decreased lung vascular endothelial growth factor (VEGF) protein levels in pups (Figure 5a,b), although combined exposure had no additive effect (Figure 5a,b). Similarly, expression of the VEGF receptor type 2 (VEGFR2) was significantly reduced in the lungs 


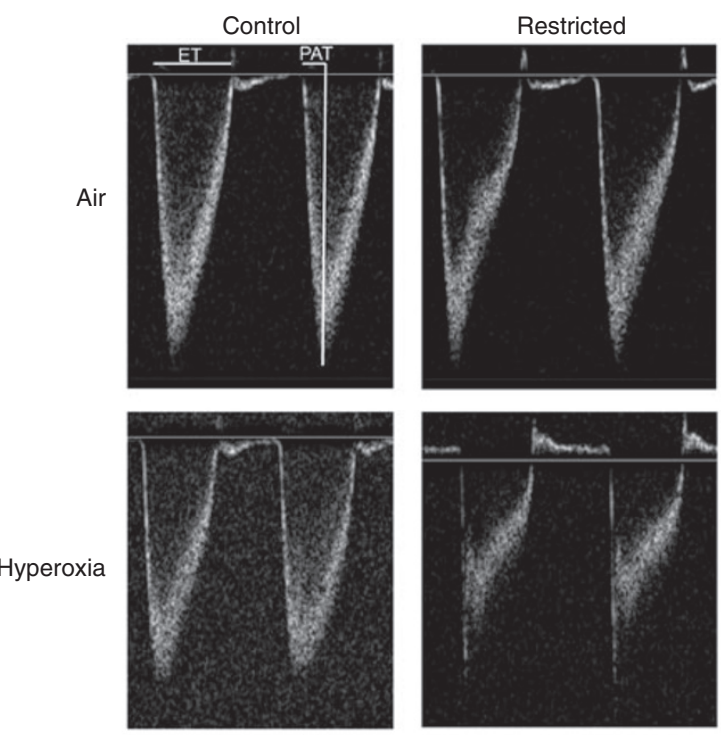

b

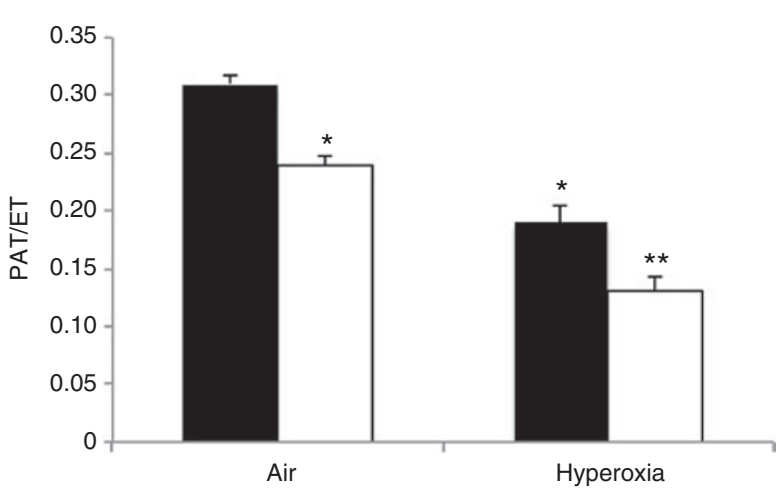

Figure 2. Postnatal growth restriction and hyperoxia induce pulmonary hypertension. (a) Pulse-wave Doppler images across the pulmonary outflow tract. Peak flow acceleration time (PAT, indicated in top left panel) decreases with increasing pulmonary arterial pressures; the ratio of PAT to total pulmonary ejection time (ET, indicated in top left panel) varies inversely with pulmonary arterial pressure. (b) PAT/ET ratios. Data from control litters are depicted by black columns; data from restricted litters are depicted by white columns. Echocardiography was performed on $N \geq 9$ animals per group. ${ }^{*} P<0.05$ vs. room air control. ${ }^{* *} P<0.05$ vs. hyperoxia control.

of postnatal growth restriction- and hyperoxia-exposed pups, with no further effect of combined exposure (Figure $5 c, d$ ).

VEGF expression is regulated by the hypoxia inducible factor (HIF) family of transcription factors (12). PNGR or hyperoxia decreased lung protein levels of HIF- $1 \alpha$ and HIF- $2 \alpha$, while combined exposure had no further effect (Figure 6a-d).

Under normoxic conditions, HIF mRNA translation is regulated by the mammalian target of rapamycin (mTOR) pathway (13). Levels of phosphorylated 4E-BP1 protein, a marker of mTOR activity, were decreased in the lungs of PNGR- and hyperoxia-exposed pups, while combined exposure had no further effect (Figure 7a,b). mTOR activity is positively regulated by the branched chain amino acids (BCAA) leucine, isoleucine, and valine (14). PNGR and hyperoxia significantly decreased lung BCAA levels, and combined exposure demonstrated an additive effect (Figure 7c).
Endothelial nitric oxide synthase (eNOS) is also regulated by HIFs and is an important driver of pulmonary vasodilation by increasing NO-induced cGMP levels (15). Lung eNOS expression was significantly decreased by PNGR or exposure to hyperoxia, while combined exposure had no further effects (Figure 8a,b). Accordingly, PNGR and hyperoxia significantly decreased total lung nitrite and nitrate levels (Figure 8c).

Figure 9 depicts the proposed signaling pathways leading to impaired lung development in postnatal growth restrictionand hyperoxia-exposed rats.

A summary of cardiopulmonary effects of PNGR that are unique to hyperoxia, similar to hyperoxia, and additive is presented in Table 1.

\section{DISCUSSION}

Following preterm birth, the infant must complete lung development from the late canalicular or early saccular stage in a relatively hyperoxic extrauterine environment, which often produces arrested parenchymal and pulmonary vascular growth (16). While PH is now recognized as a common and serious complication in preterm infants with BPD, its specific triggers are not well understood.

We found that PNGR significantly decreased body weight after $14 \mathrm{~d}$. Previous studies have shown that compared to pups in a standard litter of 10, pups in a litter of 16 have body weight that is $22 \%$ lower at $10 \mathrm{~d}$ of life and $24 \%$ lower at $20 \mathrm{~d}$ of life, and a $25 \%$ decrease in body fat at $22 \mathrm{~d}$ of life (17). While exposure to $75 \%$ oxygen from birth did not decrease body weight at day 14 , it did decrease body weight by an additional $11 \%$ in growth-restricted pups. Other investigators have shown that exposure to more extreme hyperoxia decreases body weight $(18,19)$. Our data suggest BPD could arise from oxygenmediated lung injury in combination with effects arising from oxygen-mediated growth restriction.

Perhaps even more compelling, our data suggest that PNGR may independently trigger changes in cardiac and pulmonary vascular development. We found that PNGR induced $\mathrm{PH}$ and $\mathrm{RVH}$ at $14 \mathrm{~d}$ even without exposure to hyperoxia, and amplified the effect of hyperoxia on RVH, suggesting that distinct mechanisms triggered by each insult could account for the cumulative increase. A recent study using a similar model of PNGR identified an increase in pulmonary arterial pressure and increased Fulton's index in male rats at $9 \mathrm{wk}$ of age (20). Our data indicate that increased pulmonary arterial pressure and RVH are manifest at $2 \mathrm{wk}$ of age in both males and females.

Cardiac remodeling is measured by the Fulton's index as a ratio of right and left ventricular weights. When normalized to body weight, PNGR and hyperoxia each increased right ventricular weight, with the greatest effect seen when the insults were combined. PNGR and hyperoxia also significantly decreased left ventricular + septal weight when normalized to body weight, although their combined exposure had no further effect. These data suggest that PNGR and hyperoxia may induce left ventricular hypoplasia by common mechanisms. In rats, cardiomyocytes switch from a fetal hyperplastic 
a

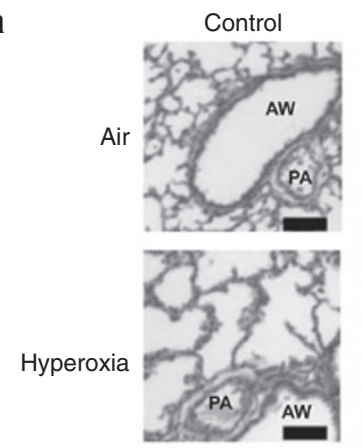

b
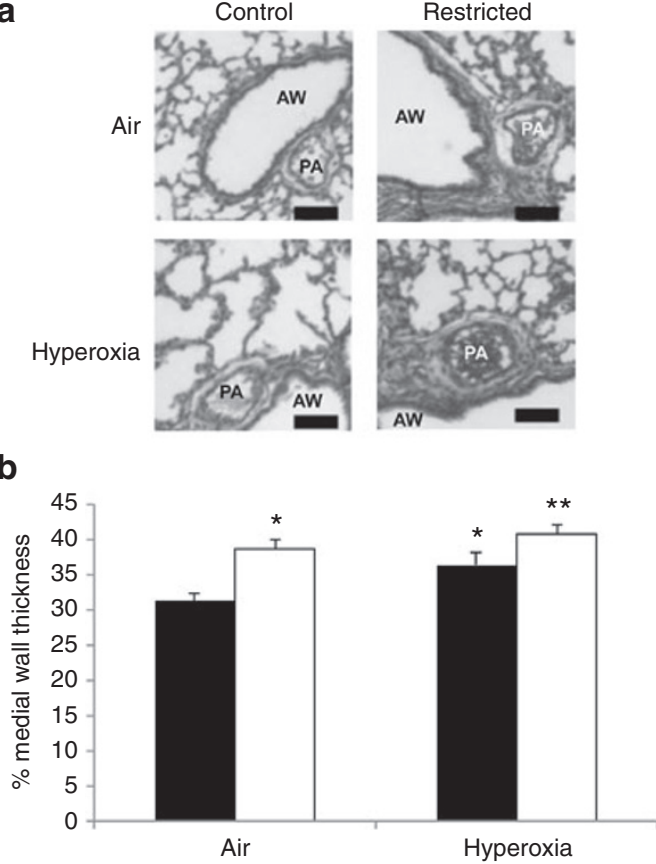
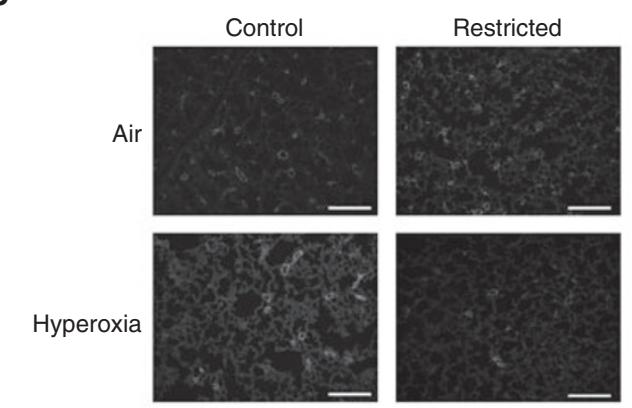

d

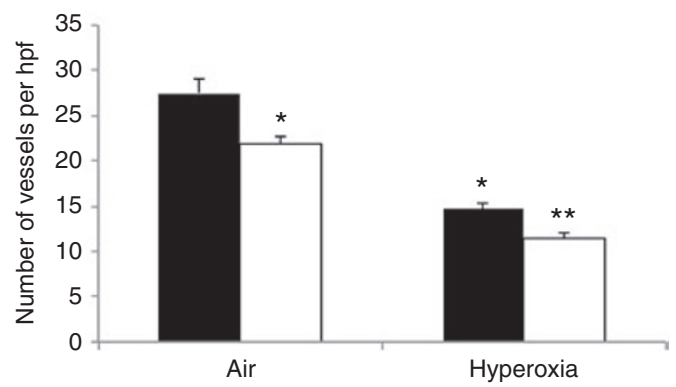

Figure 3. Postnatal growth restriction (PNGR) and hyperoxia induce pulmonary vascular remodeling. (a) Representative images of lung sections stained with H\&E captured at $\times 40$ original magnification. Scale bars (bottom right) $=20 \mu \mathrm{m}$. Pulmonary artery (PA) medial wall thickness (MWT) was calculated using ImageJ. Airways (AW) are indicated (b) PNGR and hyperoxia increased medial wall thickness. Data from control litters are depicted by black columns; data from restricted litters are depicted by white columns. MWT was calculated for $\geq 40$ vessels from $N \geq 6$ animals per group. (c) Representative images of vessels labeled for von Willebrand factor and captured at $\times 40$ original magnification. Scale bars (bottom right) $=100 \mu \mathrm{m}$. (d) PNGR and hyperoxia decreased vessel density. Data from control litters are depicted by black columns; data from restricted litters are depicted by white columns. Vessels were counted from $8-10$ images from $N \geq 6$ animals per group. ${ }^{*} P<0.05$ vs. room air control. ${ }^{* *} P<0.05$ vs. hyperoxia control.

phenotype to a neonatal hypertrophic phenotype between postnatal day 3 and 4 (21). We suggest that PNGR or hyperoxia impairs this transitional switch, resulting in decreased left ventricular weight at $14 \mathrm{~d}$. Although left ventricular mass may subsequently normalize due to accelerated hypertrophy after $14 \mathrm{~d}$, there will be fewer cardiomyocytes, which may impair cardiac function in later life. This could explain the reduced lifespan observed in neonatal mice exposed to $100 \%$ oxygen between postnatal days 1 and 4 (22). Furthermore, in human infants, preterm birth is associated with reduced end systolic and stroke volumes in young adults (23). Together, these data suggest that PNGR and hyperoxia may impede normal cardiac development and have important implications for the longterm cardiovascular health of preterm infants. Further studies using these models may help to identify the underlying mechanisms.

Hyperoxia-induced PH is characterized by increased medial wall thickness of the pulmonary arteries and decreased lung vessel density, indicating impaired angiogenesis (10). Our data indicate that PNGR affected both markers of lung vascular development and amplified the effects of oxygen. PNGR under normoxic conditions significantly increased PA medial wall thickness in male rats at $9 \mathrm{wk}$ of age (20), and here we report a significant increase in medial wall thickness at $2 \mathrm{wk}$ of age regardless of gender. In contrast, while oxygen induced a significant increase in mean alveolar area and a significant decrease in radial alveolar count, PNGR did not have any detectable effect on alveolarization under normoxic or hyperoxic conditions. BPD and PH are often assumed to result from lung injury, but our data suggest a more complex relationship. However, the factors important for normal alveolar development that are impaired by hyperoxia but not by PNGR are currently unknown.

We next monitored lung gene expression to identify the molecular pathways impeded by hyperoxia and by PNGR. We found that PNGR and hyperoxia depressed VEGF, VEGFR2, and eNOS expression, as well as their upstream mediators, HIF1/2 and mTOR. Lung VEGF expression is decreased in premature infants who die with BPD $(24,25)$, and in various rodent models of neonatal hyperoxic lung injury (26). Disruption of pulmonary VEGF leads to abnormal vascular and alveolar development, lung hypoplasia, and pulmonary hypertension (27-29), and pharmacologic inhibition of VEGF receptors in neonatal rats produces similar impairments in lung alveolarization and vascular growth $(30,31)$.

VEGF expression is regulated by the hypoxia inducible factor (HIF) family of transcription factors (12). HIF activity is rapidly downregulated by increased oxygen, but our finding that PNGR causes decreased lung HIF expression is novel. Under normoxic conditions, the mTOR pathway regulates HIF signaling by enhancing mRNA translation to a rate exceeding protein degradation $(13,32)$. Protein translation is a complex process regulated by initiation factors that control the assembly of ribosomes on mRNA. Initiation factors are 


\section{Articles | Wedgwood et al.}

a

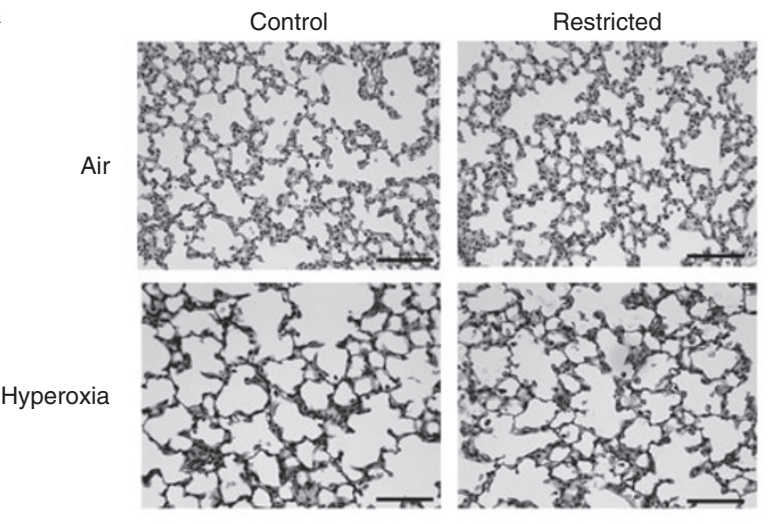

b

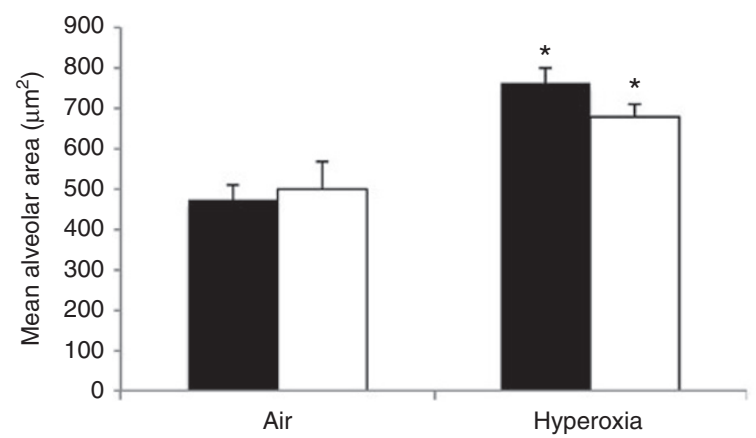

C

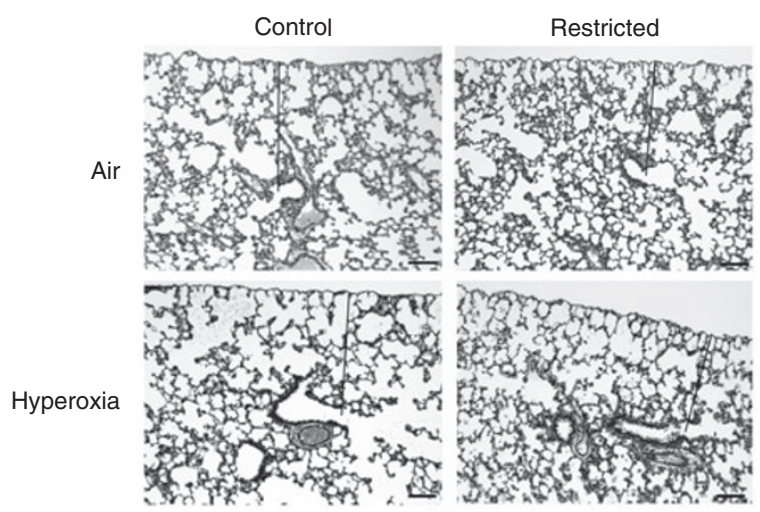

d

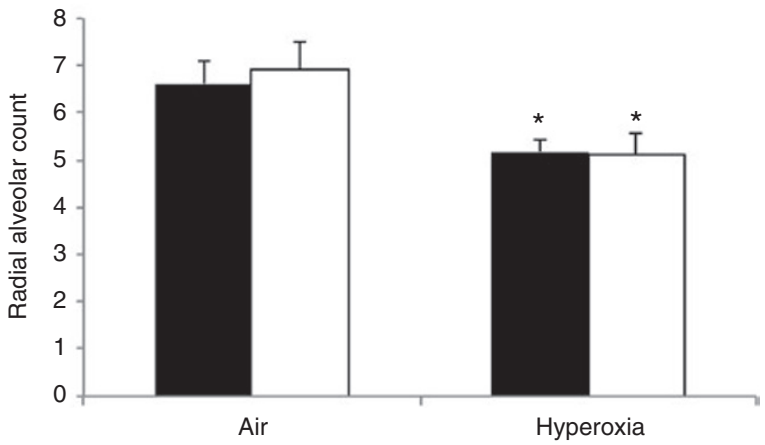

Figure 4. Hyperoxia but not postnatal growth restriction impairs alveolar development. (a) Representative images of lung sections stained with hematoxylin and captured at $\times 20$ original magnification. Scale bars (bottom right) $=100 \mu \mathrm{m}$. (b) Mean alveolar area was determined using Scion imaging software. Data from control litters are depicted by black columns; data from restricted litters are depicted by white columns. Six to eight images were captured for $N \geq 4$ animals per group. (c) Representative images of lung sections stained with hematoxylin and captured at $\times 10$ original magnification. Scale bars (bottom right) $=100 \mu \mathrm{m}$. (d) Radial alveolar counts were determined as originally described by Emery and Mithal (38). Data from control litters are depicted by black columns; data from restricted litters are depicted by white columns. Five to nine images were captured for $N \geq 6$ animals per group. ${ }^{*} P<0.05$ vs. room air control.

a

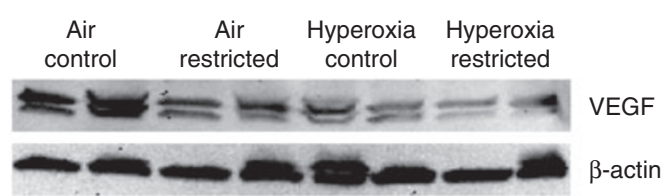

b

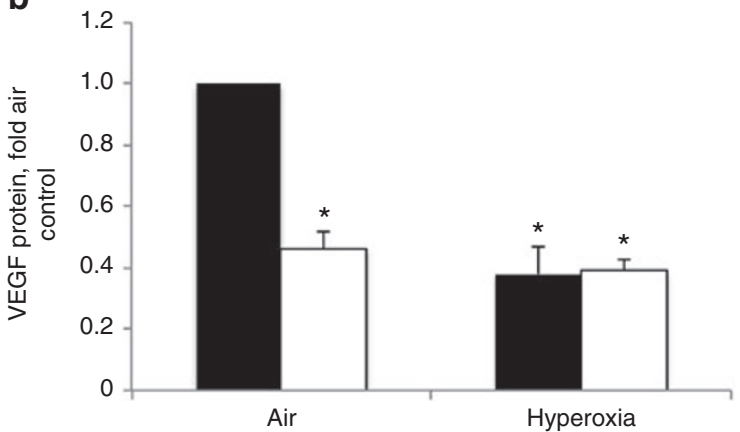

c

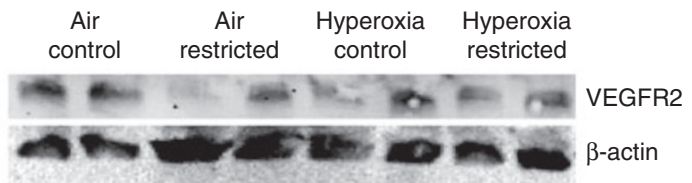

d

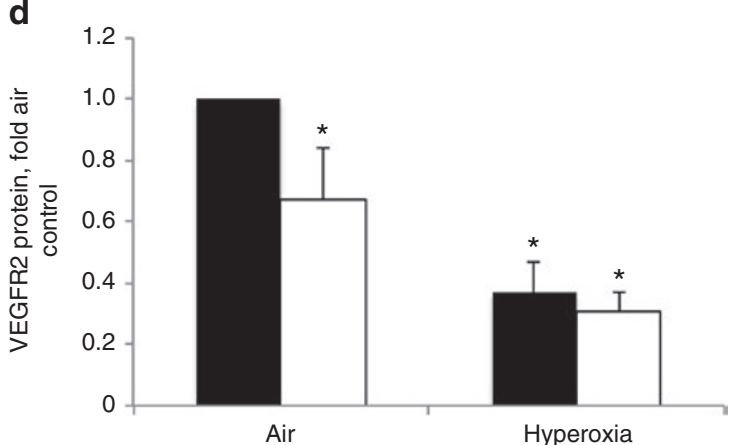

Figure 5. Postnatal growth restriction and hyperoxia decrease VEGF signaling in lung. (a) Representative Western blot of lung protein probed for VEGF and $\beta$-actin. (b) VEGF band intensities were normalized to $\beta$-actin band intensities and expressed as fold change relative to air controls. Data from control litters are depicted by black columns; data from restricted litters are depicted by white columns. (c) Representative western blot of lung protein probed for VEGFR2 and $\beta$-actin. (d) VEGFR2 band intensities were normalized to $\beta$-actin band intensities and expressed as fold change relative to air controls. Data from control litters are depicted by black columns; data from restricted litters are depicted by white columns. $N \geq 7$ animals per group. ${ }^{*} P<0.05$ vs. room air control. 
a

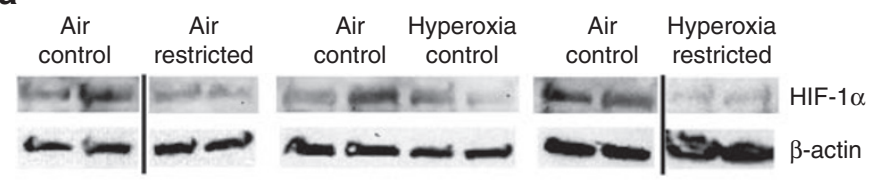

b

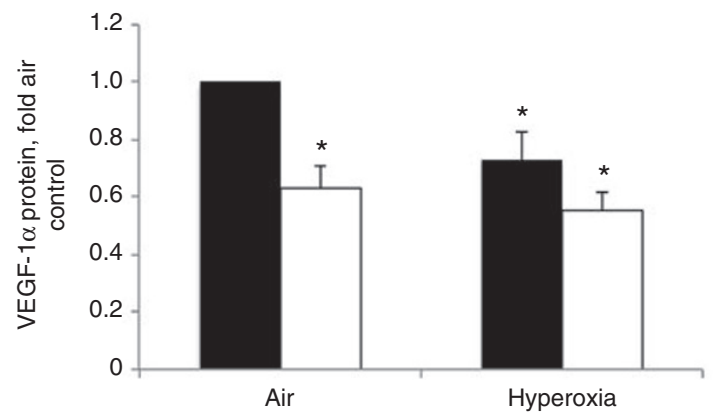

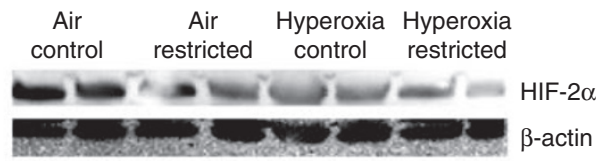

d

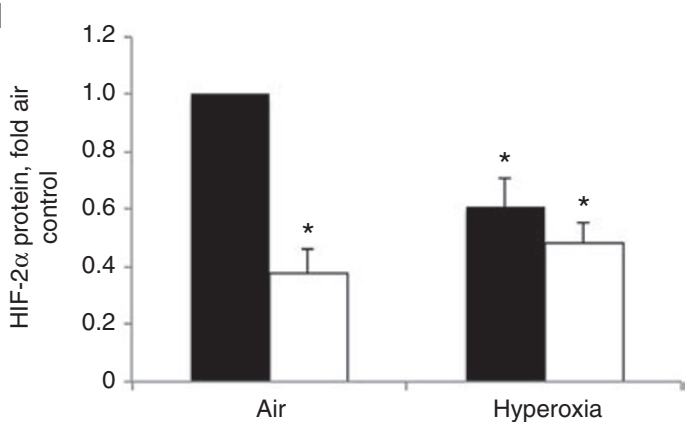

Figure 6. Postnatal growth restriction and hyperoxia decrease HIF signaling in lung. (a) Representative Western blots of lung protein probed for HIF-1 $\alpha$ and $\beta$-actin. Bands separated by a black line were from the same western blot membrane and exposure, and images were processed identically. (b) HIF-1 $\alpha$ band intensities were normalized to $\beta$-actin band intensities and expressed as fold change relative to air controls. Data from control litters are depicted by black columns; data from restricted litters are depicted by white columns. (c) Representative western blot of lung protein probed for HIF-2 $\alpha$ and $\beta$-actin. (d) HIF-2 $\alpha$ band intensities were normalized to $\beta$-actin band intensities and expressed as fold change relative to air controls. Data from control litters are depicted by black columns; data from restricted litters are depicted by white columns. $N \geq 9$ animals per group. ${ }^{*} P<0.05$ vs. room air control.

a

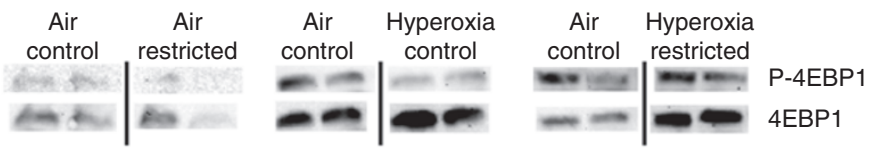

b

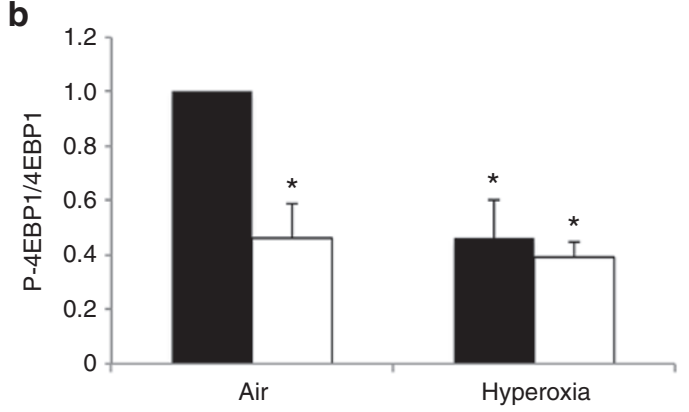

C

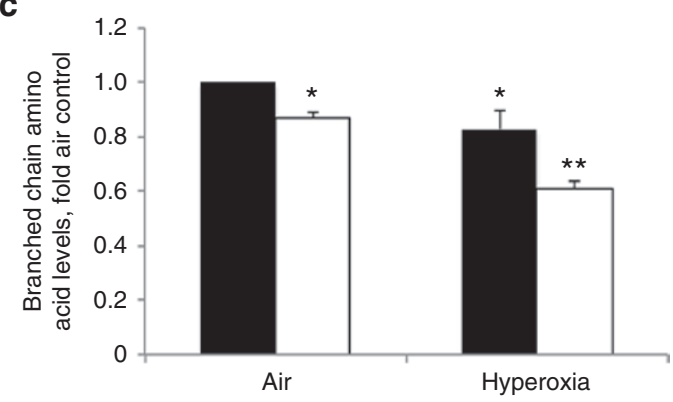

Figure 7. Postnatal growth restriction and hyperoxia decrease mTOR signaling in lung. (a) Representative Western blots of lung protein probed for phospho-4E-BP1 and 4E-BP1. Bands separated by a black line were from the same Western blot membrane and exposure, and images were processed identically. (b) Phospho-4E-BP1 band intensities were normalized to 4E-BP1 band intensities and expressed as fold change relative to air controls. Data from control litters are depicted by black columns; data from restricted litters are depicted by white columns. $N \geq 6$ animals per group. (c) Metabolomic analysis to quantify branched chain amino acids in lung. Leucine, isoleucine, and valine levels were quantified and expressed relative to air controls, and the combined fold change of branched chain amino acids determined. Data from control litters are depicted by black columns; data from restricted litters are depicted by white columns. $N=4$ animals per group. ${ }^{*} P<0.05$ vs. room air control. ${ }^{* *} P<0.05$ vs. hyperoxia control.

regulated by repressor proteins including 4E-BP1, and mTOR signaling promotes protein translation, in part, by phosphorylating and inactivating 4E-BP1. Therefore, we monitored $4 \mathrm{E}-\mathrm{BP} 1$ protein phosphorylation as a marker of mTOR activity, and found a significant decrease in response to postnatal growth restriction and to hyperoxia. Little is known about the effect of hyperoxia on mTOR signaling, although human infant lung fibroblasts exposed to $95 \% \mathrm{O}_{2}$ showed diminished protein synthesis together with decreased phosphorylation of 4E-BP1 (33).
Multiple factors regulate mTOR signaling including insulinlike growth factor-1 (IGF-1) (13). Interestingly, PNGR pups display decreases in serum IGF-1 and increased glucose tolerance $(17,34)$, while IGF- 1 protein is decreased in the lungs of neonatal rats exposed to $95 \% \mathrm{O}_{2}$ for $72 \mathrm{~h}$ (35). Additional studies are required to determine potential roles for IGF-1 in PNGR- and hyperoxia-induced $\mathrm{PH}$.

mTOR activity is positively regulated by the BCAA leucine, isoleucine, and valine (14). Using metabolomics analysis of lung tissue, we identified a significant decrease in BCAA levels 


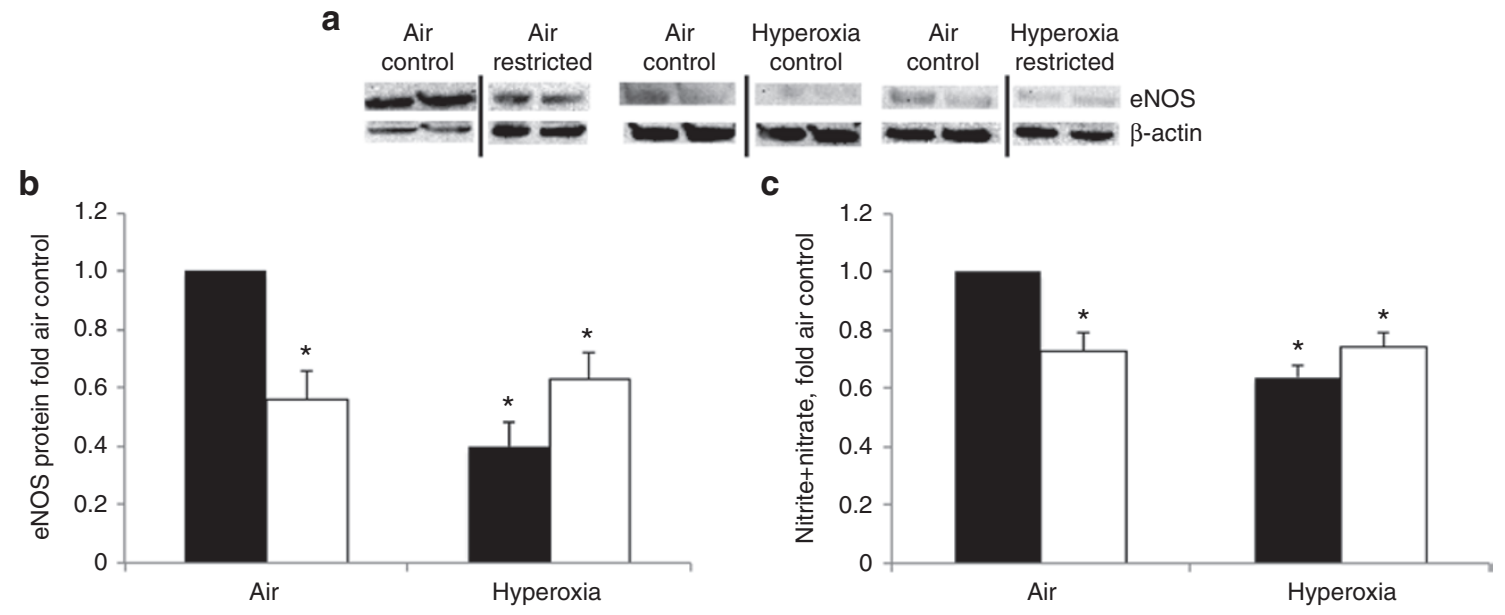

Figure 8. Postnatal growth restriction and hyperoxia decrease eNOS expression and nitrate/nitrite levels in lung. (a) Representative western blots of lung protein probed for eNOS and $\beta$-actin. Bands separated by a black line were from the same western blot membrane and exposure, and images were processed identically. (b) eNOS band intensities were normalized to $\beta$-actin band intensities and expressed as fold change relative to air controls. Data from control litters are depicted by black columns; data from restricted litters are depicted by white columns. (c) Lung NOS metabolites nitrate and nitrite were quantified using a kit and expressed relative to air controls. Data from control litters are depicted by black columns; data from restricted litters are depicted by white columns. ${ }^{*} P<0.05$ vs. room air control.

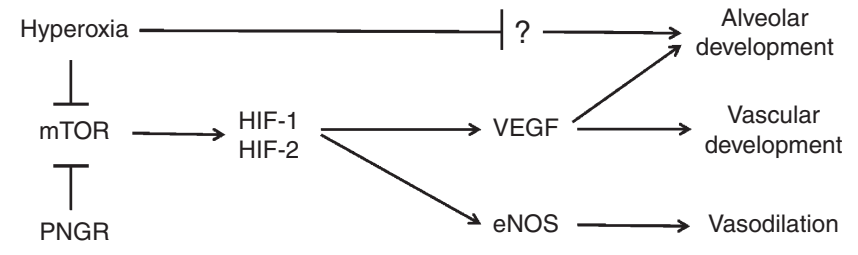

Figure 9. Proposed pathways of postnatal growth restriction (PNGR)- and hyperoxia-impaired lung development. PNGR and hyperoxia attenuate mTOR activity, resulting in decreased expression of HIF target genes including VEGF and eNOS. Decreased eNOS expression impairs pulmonary vasodilation and decreased VEGF expression impairs vascular and alveolar development. Additional unknown factors (depicted by "?") are essential for alveolar development; hyperoxia, but not PNGR, impairs these factors.

in both the PNGR and hyperoxia groups, with a further significant reduction in the combined group. Of the nine amino acids essential for human nutrition (i.e., must be supplied in the diet), the three BCAAs and lysine were altered by both insults while the five other essential amino acids were not affected (data not shown). While the mechanisms that selectively decrease lung BCAA levels must still be identified, our data raise the possibility that BCAA supplementation may attenuate the effects of PNGR and/or hyperoxia in neonatal rats.

Endothelial NOS (eNOS) is regarded as the most important regulator of nitric oxide $(\mathrm{NO})$ production in the perinatal lung vasculature, and its disruption affects lung alveolarization and angiogenesis. We found decreased lung eNOS expression and NO metabolites (nitrite and nitrate, Figure $8 \mathbf{a}-\mathrm{c}$ ) in response to PNGR and to hyperoxia. eNOS expression, together with VEGF and VEGFR2 expression, vessel density and radial alveolar counts, were likewise decreased in a fetal sheep model of fetal growth restriction (36).

It is likely that factors other than just nutrient and energy intake contribute the poor growth in this model (e.g., higher thermoregulatory energy expenditure) (11). In more recent
Table 1. Summary of similarities and differences between hyperoxia and PNGR in neonatal rat pups

\begin{tabular}{lccc}
\hline & Hyperoxia & PNGR & Hyperoxia + PNGR \\
\hline Additive effects & & & \\
Fulton's index & $\uparrow$ & $\uparrow$ & $\uparrow \uparrow$ \\
RV weight/body & $\uparrow$ & $\uparrow$ & $\uparrow \uparrow$ \\
weight & & & \\
Medial wall thickness & $\uparrow$ & $\uparrow$ & $\uparrow \uparrow$ \\
Vessels per HPF & $\downarrow$ & $\downarrow$ & $\downarrow \downarrow$ \\
BCAA & $\downarrow$ & $\downarrow$ & $\downarrow \downarrow$
\end{tabular}

Effects unique to hyperoxia

$\begin{array}{llll}\text { Mean alveolar area } & \uparrow & - & \uparrow \\ \text { Radial alveolar count } & \downarrow & - & \downarrow\end{array}$

Effects similar but not additive

$\begin{array}{lccc}\text { VEGF/VEGFR2 } & \downarrow & \downarrow & \downarrow \\ \text { HIF1 } \alpha / \text { HIF2 } \alpha & \downarrow & \downarrow & \downarrow \\ \text { P-4E-BP1 } & \downarrow & \downarrow & \downarrow \\ \text { eNOS } & \downarrow & \downarrow & \downarrow \\ \text { Nitrate+nitrite } & \downarrow & \downarrow & \downarrow\end{array}$

BCAA, branched chain amino acids; eNOS, endothelial nitric oxide synthase; HPF, highpower field; PNGR, postnatal growth restriction; RV, right ventricle.

studies pups in a litter size of 16 had reduced growth, altered body composition, increased glucose tolerance, and poor neurodevelopment relative to pups from a litter size of $10(17,34)$. It will be important to determine whether these additional factors play a mechanistic role in cardiac and pulmonary vascular development, and whether they are applicable to infants born prematurely.

In summary, our data suggest that PNGR induces $\mathrm{PH}$, right ventricular and pulmonary vascular remodeling, and augments the effects of oxygen in neonatal rats with BPD and PH. 
Potential mechanisms include impaired mTOR/HIF/VEGF signaling and impaired NO-mediated vasodilation (Figure 9). This novel rodent model may be clinically relevant and allow for investigation of mechanisms that induce $\mathrm{PH}$ in lowbirth-weight preterm infants. PNGR is commonplace in sick preterm neonates, and further study focused on specific dietary targets could provide paradigm-shifting insights into the prevention of $\mathrm{BPD}$ and $\mathrm{PH}$.

\section{METHODS}

\section{Animals and Echocardiography}

The animal protocol was approved by the Institutional Animal Care and Use Committee at UC Davis. Timed-pregnant Sprague Dawley dams at E14-E16 were ordered from Harlan Laboratories (Indianapolis, IN). Rats were housed in plastic cages with a $12 \mathrm{~h}$ dark:light cycle and allowed to feed ad libitum with a standard diet (2018 Teklad from Harlan). After birth, pups were pooled and randomly assigned to litters of 10 pups (control) or 17 pups (growth-restricted). Additionally, pups were randomly assigned to cages maintained in room air or exposed to $75 \%$ oxygen in a Plexiglas chamber (Biospherix, Lacona, NY) continuously, and dams were rotated with the appropriate control or growth-restricted dam every $24 \mathrm{~h}$. At postnatal day 14 , the pups were analyzed by echocardiography (37) using a VisualSonics Vevo 2100 in vivo ultrasound imaging system (VisualSonics, Toronto, Ontario, Canada). Pups were then weighed and euthanized for tissue harvest. Hearts and lungs were snap-frozen in liquid nitrogen and stored at $-80{ }^{\circ} \mathrm{C}$, or lungs were inflation-fixed with $4 \%$ formalin under $25 \mathrm{~cm} \mathrm{H}_{2} \mathrm{O}$ pressure.

\section{Measurement of Right Ventricular Hypertrophy (RVH)}

Fulton's index (the weight of the right ventricle (RV) divided by the weight of the left ventricle (LV) + septum) was determined to assess $\mathrm{RVH}$. Additionally, RV and LV + septum weights were normalized to body weight (10).

\section{Lung Vascular and Morphometric Analysis}

Images of $5 \mu \mathrm{m}$ sections from inflation-fixed lungs were captured using an Olympus BH2-RFCA fluorescence microscope and a Leica DFC 500 camera. All analyses were performed in a blinded fashion. To determine average vessel number, immunofluorescence was performed using a von Willebrand primary antibody (1:50, Dako, Carpinteria, CA) and a rhodamine red secondary antibody (Molecular Probes, Carlsbad, CA). Eight to ten images of pulmonary vessels approximately $10-100 \mu \mathrm{m}$ in diameter were counted and averaged for each slide and reported as the number of vessels per highpower field. To determine medial wall thickness, hematoxylin and eosin-stained lung sections were imaged at $40 \times$ magnification. Eight to ten images from each sample were analyzed and medial wall thickness of small pulmonary arteries (50-100 $\mu \mathrm{m}$ diameter) determined using Image J software (National Institutes of Health, Bethesda, MD). medial wall thickness was defined as the area of the PA wall divided by the total area of the vessel. For alveolar morphometry, sections were stained with hematoxylin overnight. Six to eight images were acquired from each slide at $20 \times$ magnification. Images were analyzed by a single operator using Scion Image software (Scion, Frederick, MD). Mean alveolar area was determined for each image and averaged to give a composite value for each animal. Radial alveolar counts were determined from five to nine images acquired at $10 \times$ magnification as described by Emery and Mithal (38).

\section{Western Blotting}

Lung tissue was suspended in radioimmunoprecipitation assay (RIPA) buffer containing protease and phosphatase inhibitors and sonicated on ice. Protein content was determined by the Bradford method and western blotting performed as described previously (39). Blots were probed using primary antibodies against VEGF (ab46154, 1:1,000, Abcam, Cambridge, MA), VEGFR2 (2479S, 1:1,000, Cell Signaling Technology, Danvers, MA), HIF-1á and HIF-2á (NB100105 and NB100-132SS2 respectively, 1:1,000, Novus Biologicals,
Littleton, CO), phospho-4E-BP1 and 4E-BP1 (2855S and 9452S respectively, 1:250, Cell Signaling Technology), eNOS (610296, 1:1000, BD Transduction Laboratories, San Jose, CA), and â-actin (ab6276, 1:4,000, Abcam) at $4{ }^{\circ} \mathrm{C}$ overnight followed by a $60 \mathrm{~min}$ incubation with the appropriate secondary antibody at room temperature. Chemiluminescence generated by Super Signal West Femto substrate (Thermo Fisher Scientific) was detected and quantified using a Kodak Image Station and software. Signals were normalized to $\hat{a}$-actin and expressed as fold change relative to air controls.

\section{Metabolomic Analysis of Branched Chain Amino Acids}

Lung tissue was submitted to the West Coast Metabolomics Center at UC Davis to quantify the concentrations of leucine, isoleucine, and valine. Lung samples $(5 \mu \mathrm{g})$, stored at $-80^{\circ} \mathrm{C}$, were thawed on ice, extracted, derivatized and metabolite levels were quantified by gas chromatography time-of-flight mass spectrometry as previously described (40). Briefly, output results were filtered based on multiple parameters to exclude noisy or inconsistent peaks. All entries in BinBase were matched against the Fiehn mass spectral library of 1,200 authentic metabolite spectra using retention index and mass spectrum information or the NIST11 commercial library. Data, reported as quantitative ion peak heights, were normalized by the sum intensity of all annotated metabolites and used for further statistical analysis. Fold change of leucine, isoleucine, and valine concentrations relative to air controls were combined to determine the fold change in branched chain amino acids in each group.

\section{NOS Activity}

Nitrite and nitrate levels were determined in lung homogenates as an indirect marker of NOS activity. $100 \mu \mathrm{g}$ protein samples were assayed in duplicate using a kit (Oxford Biomedical Research, Rochester Hills, $\mathrm{MI})$ according to the manufacturers' instructions. Nitrite and nitrate levels were expressed relative to air controls.

\section{Statistical Analysis}

Data are presented as means \pm SEM and further expressed as fold change relative to air controls where indicated. "N" represents the number of animals in each group. The effects of PNGR and hyperoxia were tested by two-way ANOVA (GraphPad Software, La Jolla, CA). A significant interaction between PNGR and hyperoxia was not detected in any analysis, and the effect of each independent variable was tested from the two-way ANOVA. If the F-test was significant, a Bonferroni post hoc test was performed. The independent variables were considered significant at $P<0.05$.

\section{SUPPLEMENTARY MATERIAL}

Supplementary material is linked to the online version of the paper at http://www.nature.com/pr

\section{ACKNOWLEDGMENTS}

We thank Johannes Fahrmann, Michael La Frano, Oliver Fiehn, and John Newman at the West Coast Metabolomics Center for the Metabolomics analysis.

\section{STATEMENT OF FINANCIAL SUPPORT}

This project was made possible in part by support from: grant CMNSW13, Children's Miracle Network, Sacramento, CA; grant DK097154, National Institutes of Health West Coast Metabolomics Center, Davis, CA; grant OD010389 National Institutes of Health S10 shared equipment grant.

Disclosure: The authors have are no disclosures to report.

\section{REFERENCES}

1. Doyle LW, Anderson PJ. Long-term outcomes of bronchopulmonary dysplasia. Semin Fetal Neonatal Med 2009;14:391-5.

2. Slaughter JL, Pakrashi T, Jones DE, South AP, Shah TA. Echocardiographic detection of pulmonary hypertension in extremely low birth weight infants with bronchopulmonary dysplasia requiring prolonged positive pressure ventilation. J Perinatol 2011;31:635-40.

3. Khemani E, McElhinney DB, Rhein L, et al. Pulmonary artery hypertension in formerly premature infants with bronchopulmonary dysplasia: clinical features and outcomes in the surfactant era. Pediatrics 2007;120: 1260-9. 
4. Berkelhamer SK, Mestan KK, Steinhorn RH. Pulmonary hypertension in bronchopulmonary dysplasia. Semin Perinatol 2013;37:124-31.

5. Bhat R, Salas AA, Foster C, Carlo WA, Ambalavanan N. Prospective analysis of pulmonary hypertension in extremely low birth weight infants. Pediatrics 2012;129:e682-9.

6. Check J, Gotteiner N, Liu X, et al. Fetal growth restriction and pulmonary hypertension in premature infants with bronchopulmonary dysplasia. J Perinatol 2013;33:553-7.

7. Ehrenkranz RA, Younes N, Lemons JA, et al. Longitudinal growth of hospitalized very low birth weight infants. Pediatrics 1999;104(2 Pt 1): $280-9$.

8. Cooke RJ, Ainsworth SB, Fenton AC. Postnatal growth retardation: a universal problem in preterm infants. Arch Dis Child Fetal Neonatal Ed 2004;89:F428-30.

9. Amy RW, Bowes D, Burri PH, Haines J, Thurlbeck WM. Postnatal growth of the mouse lung. J Anat 1977;124(Pt 1):131-51.

10. Ladha F, Bonnet S, Eaton F, Hashimoto K, Korbutt G, Thébaud B. Sildenafil improves alveolar growth and pulmonary hypertension in hyperoxiainduced lung injury. Am J Respir Crit Care Med 2005;172:750-6.

11. Fiorotto ML, Burrin DG, Perez M, Reeds PJ. Intake and use of milk nutrients by rat pups suckled in small, medium, or large litters. Am J Physiol 1991;260(6 Pt 2):R1104-13.

12. Rajatapiti P, van der Horst IW, de Rooij JD, et al. Expression of hypoxiainducible factors in normal human lung development. Pediatr Dev Pathol 2008;11:193-9.

13. Fukuda R, Hirota K, Fan F, Jung YD, Ellis LM, Semenza GL. Insulin-like growth factor 1 induces hypoxia-inducible factor 1-mediated vascular endothelial growth factor expression, which is dependent on MAP kinase and phosphatidylinositol 3-kinase signaling in colon cancer cells. J Biol Chem 2002;277:38205-11.

14. Anthony JC, Yoshizawa F, Anthony TG, Vary TC, Jefferson LS, Kimball SR. Leucine stimulates translation initiation in skeletal muscle of postabsorptive rats via a rapamycin-sensitive pathway. J Nutr 2000;130: 2413-9.

15. Coulet F, Nadaud S, Agrapart M, Soubrier F. Identification of hypoxiaresponse element in the human endothelial nitric-oxide synthase gene promoter. J Biol Chem 2003;278:46230-40.

16. Thébaud B, Abman SH. Bronchopulmonary dysplasia: where have all the vessels gone? Roles of angiogenic growth factors in chronic lung disease. Am J Respir Crit Care Med 2007;175:978-85.

17. Jou MY, Lönnerdal B, Griffin IJ. Effects of early postnatal growth restriction and subsequent catch-up growth on body composition, insulin sensitivity, and behavior in neonatal rats. Pediatr Res 2013;73:596-601.

18. Visser YP, Walther FJ, Laghmani el H, Laarse Av, Wagenaar GT. Apelin attenuates hyperoxic lung and heart injury in neonatal rats. Am J Respir Crit Care Med 2010;182:1239-50.

19. Sharma D, Nkembi AS, Aubry E, et al. Maternal PUFA $\omega-3$ supplementation prevents neonatal lung injuries induced by hyperoxia in newborn rats. Int J Mol Sci 2015;16:22081-93.

20. Zhang L, Tang L, Wei J, et al. Extrauterine growth restriction on pulmonary vascular endothelial dysfunction in adult male rats: the role of epigenetic mechanisms. J Hypertens 2014;32:2188-98; discussion 2198.

21. Li F, Wang X, Capasso JM, Gerdes AM. Rapid transition of cardiac myocytes from hyperplasia to hypertrophy during postnatal development. J Mol Cell Cardiol 1996;28:1737-46.

22. Yee M, White RJ, Awad HA, Bates WA, McGrath-Morrow SA, O’Reilly MA. Neonatal hyperoxia causes pulmonary vascular disease and shortens life span in aging mice. Am J Pathol 2011;178:2601-10.
23. Lewandowski AJ, Augustine D, Lamata P, et al. Preterm heart in adult life: cardiovascular magnetic resonance reveals distinct differences in left ventricular mass, geometry, and function. Circulation 2013;127:197-206.

24. Bhatt AJ, Pryhuber GS, Huyck H, Watkins RH, Metlay LA, Maniscalco WM. Disrupted pulmonary vasculature and decreased vascular endothelial growth factor, Flt-1, and TIE-2 in human infants dying with bronchopulmonary dysplasia. Am J Respir Crit Care Med 2001;164(10 Pt 1):1971-80.

25. Abman SH. Impaired vascular endothelial growth factor signaling in the pathogenesis of neonatal pulmonary vascular disease. Adv Exp Med Biol 2010;661:323-35.

26. Hosford GE, Olson DM. Effects of hyperoxia on VEGF, its receptors, and HIF-2alpha in the newborn rat lung. Am J Physiol Lung Cell Mol Physiol 2003;285:L161-8.

27. Gien J, Seedorf GJ, Balasubramaniam V, Markham N, Abman SH. Intrauterine pulmonary hypertension impairs angiogenesis in vitro: role of vascular endothelial growth factor nitric oxide signaling. Am J Respir Crit Care Med 2007;176:1146-53.

28. Compernolle V, Brusselmans K, Acker T, et al. Loss of HIF-2alpha and inhibition of VEGF impair fetal lung maturation, whereas treatment with VEGF prevents fatal respiratory distress in premature mice. Nat Med 2002;8:702-10.

29. Grover TR, Parker TA, Zenge JP, Markham NE, Kinsella JP, Abman SH. Intrauterine hypertension decreases lung VEGF expression and VEGF inhibition causes pulmonary hypertension in the ovine fetus. Am J Physiol Lung Cell Mol Physiol 2003;284:L508-17.

30. Jakkula M, Le Cras TD, Gebb S, et al. Inhibition of angiogenesis decreases alveolarization in the developing rat lung. Am J Physiol Lung Cell Mol Physiol 2000;279:L600-7.

31. Le Cras TD, Markham NE, Tuder RM, Voelkel NF, Abman SH. Treatment of newborn rats with a VEGF receptor inhibitor causes pulmonary hypertension and abnormal lung structure. Am J Physiol Lung Cell Mol Physiol 2002;283:L555-62.

32. Saji M, Ringel MD. The PI3K-Akt-mTOR pathway in initiation and progression of thyroid tumors. Mol Cell Endocrinol 2010;321:20-8.

33. Shenberger JS, Myers JL, Zimmer SG, Powell RJ, Barchowsky A. Hyperoxia alters the expression and phosphorylation of multiple factors regulating translation initiation. Am J Physiol Lung Cell Mol Physiol 2005;288:L442-9.

34. Alexeev EE, Lönnerdal B, Griffin IJ. Effects of postnatal growth restriction and subsequent catch-up growth on neurodevelopment and glucose homeostasis in rats. BMC Physiol 2015;15:3.

35. Chetty A, Nielsen HC. Regulation of cell proliferation by insulin-like growth factor 1 in hyperoxia-exposed neonatal rat lung. Mol Genet Metab 2002;75:265-75.

36. Rozance PJ, Seedorf GJ, Brown A, et al. Intrauterine growth restriction decreases pulmonary alveolar and vessel growth and causes pulmonary artery endothelial cell dysfunction in vitro in fetal sheep. Am J Physiol Lung Cell Mol Physiol 2011;301:L860-71.

37. Thibault HB, Kurtz B, Raher MJ, et al. Noninvasive assessment of murine pulmonary arterial pressure: validation and application to models of pulmonary hypertension. Circ Cardiovasc Imaging 2010;3:157-63.

38. Cooney TP, Thurlbeck WM. The radial alveolar count method of Emery and Mithal: a reappraisal 1-postnatal lung growth. Thorax 1982;37:572-9.

39. Wedgwood S, McMullan DM, Bekker JM, Fineman JR, Black SM. Role for endothelin-1-induced superoxide and peroxynitrite production in rebound pulmonary hypertension associated with inhaled nitric oxide therapy. Circ Res 2001;89:357-64.

40. Fiehn O, Wohlgemuth G, Scholz M, et al. Quality control for plant metabolomics: reporting MSI-compliant studies. Plant J 2008;53:691-704. 